\title{
Increased expression of neuropilin 1 is associated with epithelial ovarian carcinoma
}

\author{
HAIYAN JIANG ${ }^{1 *}$, QINGHUA XI ${ }^{1 *}$, FEIRAN WANG ${ }^{2}$, ZHICHAO SUN $^{3}$, ZHONGWEI HUANG $^{4}$ and LEI QI ${ }^{4}$ \\ Departments of ${ }^{1}$ Obstetrics and Gynecology and ${ }^{2}$ Surgery, Affiliated Hospital of Nantong University, Nantong, Jiangsu 226001; \\ ${ }^{3}$ Medical College, Nantong University, Nantong, Jiangsu 226019; ${ }^{4}$ Department of Emergency Medicine, \\ Affiliated Hospital of Nantong University, Nantong, Jiangsu 226001, P.R. China
}

Received June 22, 2014; Accepted March 10, 2015

DOI: $10.3892 / \mathrm{mmr} .2015 .3580$

\begin{abstract}
Neuropilin 1 (NRP1) is a transmembrane glycoprotein, which interacts with vascular endothelial growth factor to prevent tumor cell apoptosis and to regulate angiogenesis. However, the precise role of NRP1 in epithelial ovarian carcinoma (EOC) remains to be elucidated. The present study aimed to determine the association between NRP1 and EOC. The expression of NRP1 in ovarian cancer and normal ovarian epithelial tissues was investigated by immunofluorescence, reverse transcription-quantitative polymerase chain reaction (RT-qPCR) and western blotting. The association between the expression of NRP1 with the development of ovarian cancer, clinicopathological characteristics and survival were also analyzed. The results from immunofluorescence, RT-qPCR and western blot analysis demonstrated that NRP1 exhibited significant upregulation in EOC compared with normal ovarian epithelial specimens $(\mathrm{P}<0.05)$. The positive expression of NRP1 was higher in cancer tissues at an advanced International Federation of Gynecology and Obstetrics stage, and in cancer tissues with lymph node metastasis and distant metastasis compared with that in cancer tissues without lymph node or distant metastasis $(\mathrm{P}<0.05)$. Higher NRP1 expression strongly predicted a shorter survival time $(\mathrm{P}<0.001)$. The present findings suggested that increased NRP1 expression may be associated with the development of EOC. Therefore, NRP1 could be used as a valuable prognostic marker as well as a potential molecular therapy target for ovarian cancer patients.
\end{abstract}

Correspondence to: Professor Zhongwei Huang and Mr. Lei Qi, Department of Emergency Medicine, Affiliated Hospital of Nantong University, 20 West Temple Road, Nantong, Jiangsu 226001, P.R. China

E-mail: tdfyhuangzw@163.com

E-mail: ntqilei@163.com

*Contributed equally

Key words: neuropilin 1, ovary, epithelial carcinoma

\section{Introduction}

Epithelial ovarian carcinoma (EOC) is the fifth leading cause of cancer-associated mortality and remains one of the most aggressive tumors of all types of gynecological malignancy in Western countries (1). According to Cancer Statistics 2012, there is an estimated 15,500 mortalities due to this disease in the United States every year (2). The majority of patients with EOC have advanced intraperitoneal metastases at diagnosis since this carcinoma frequently remains clinically silent (3). Since the treatment strategy consisting of maximum cytoreductive surgery followed by taxane plus platinum chemotherapy was established, the short-term prognosis of patients with EOC has largely improved (4). However, despite the comparatively high-level sensitivity of EOC to paclitaxel, the prognosis of advanced or recurrent cases remains poor as the majority of cases of mortality are the result of metastasis that is refractory to these chemotherapeutic agents. Although various additional molecular-targeted therapies, including anti-angiogenic agents, have been investigated in order to overcome paclitaxel resistance, the effect of such treatment is not satisfactory $(5,6)$. Currently, numerous studies have been conducted in order to identify new strategies and targets to treat this disease (7-9).

Recently, a growing emphasis has been placed on the association between the nervous system and cancer as increasing evidence supports the theory that there are common genetic mechanisms in the development of cancer and in the progression of neurodegenerative disease (10-12). The nervous system may potentially affect the development of cancer; environmental enrichment has been demonstrated to significantly inhibit xenograft tumor growth, however, the underlying mechanism remains to be elucidated (13). The neuropilin 1 (NRP1) and the neuropilin 2 (NRP2) receptors were characterized as receptors for axon guidance factors of the class-3 semaphorin (sema3) family (14). It was subsequently established that neuropilins are also expressed by endothelial cells and by numerous types of cancer cells (15), and that they are involved in the transduction of pro-angiogenic signals induced by angiogenic factors, including vascular endothelial growth factor (VEGF) and hepatocyte growth factor/scatter factor (16-18). However, the role of NRP1 in EOC remains to be elucidated. Therefore, the present study focused on the expression of NRP1 in ovarian cancer in order to determine 
whether the expression of NRP1 is associated with ovarian cancer.

\section{Patients and methods}

Patient information and tissue sampling. In total, 125 specimens of EOC from patients diagnosed between 2000 and 2010 were obtained from surgery from the Department of Pathology at the Affiliated Hospital of Nantong University (Nantong, China). None of the patients had received any form of tumor-specific therapy prior to surgery. Samples were collected from patients aged between 33 and 82 years old with a median age of 59 years. According to the classification 2009 of the International Federation of Gynecology and Obstetrics (FIGO), there were 20 cases of Stage I, 39 cases of Stage II, 35 cases of Stage III and 37 cases of Stage IV. The histological grade of the tumor was classified as GI (well differentiated) in 55 cases, GII (moderately differentiated) in 32 cases and GIII (poorly differentiated) in 38 cases. Of all the samples, there were 72 cases with lymph node metastases (median age 55 years; range $39-75$ years), 58 cases with pelvic metastases (median age 53 years; range $42-79$ years) and 53 cases with peritoneal metastases (median age 56; range 35-68 years). The follow-up period ranged between 2 and 60 months with an average cycle period of 29.7 months and a median period of 20 months. A total of 15 cases of normal ovarian epithelium specimens were obtained from preventive excision of the uterus and adnexa uteri. All tissues were obtained with the consent of the patients. The study protocol followed the guidelines of the Helsinki Declaration and was approved by the Ethics Committee (Institutional Review Board) of Nantong University.

Double-labeling immunofluorescence staining and confocal microscopy. All specimens were embedded in optimum cutting temperature and frozen in 2-methylbutane cooled by liquid nitrogen. They were then sectioned into $20 \mu \mathrm{m}$ thick sections using a cryostat. Sections were fixed with cold acetone, blocked with $10 \%$ bovine serum albumin (BSA) in phosphate-buffered saline (PBS) containing $0.2 \%$ Triton X-100 and further permeabilized/blocked in the blocking solution (5\% BSA and $0.3 \%$ Triton X-100) for $1 \mathrm{~h}$ at room temperature. Non-specific binding was blocked with $10 \%$ BSA for $30 \mathrm{~min}$. Sections were then probed with a rabbit monoclonal NRP1 primary antibody (1:100; cat. no. ab81321; Santa Cruz Biotechnology, Inc., Santa Cruz, CA, USA) overnight at $4^{\circ} \mathrm{C}$, followed by fluorescein isothiocyanate-conjugated rabbit anti-goat $\operatorname{IgG}(\mathrm{H} \& \mathrm{~L})$ secondary antibody (1:100; Abnova Corporation, Tapei, Taiwan; cat. no. PAB10575) for $2 \mathrm{~h}$ in a humidified chamber with minimal exposure to light. 4',6-diamidino-2-phenylindole (DAPI; Sigma-Aldrich, St. Louis, MO, USA) was used to visualize nuclei and sections were washed with 1X PBS. Images of the samples were captured using a confocal microscope (Leica Microsystems, Wetzlar, Germany). Sections were analyzed using a Leica SP5 high-speed spectral confocal laser-scanning microscope (Leica Microsystems) or a Zeiss LSM 710 confocal microscope (Carl Zeiss, Oberkochen, Germany). Immunofluorescence staining for single or double contractile markers was performed using randomly selected slides (4-5 slides) containing four sections per slide and examined under the confocal microscope. Specific fluorescence was captured by confocal microscopy with exposure time kept constant across all the images. Immunoreactivity was evaluated by semi-quantitative evaluation using the immunofluorescence staining intensity score and distribution score.

Evaluation of immunoreactivity. Immunoreactivity was evaluated by the quantification and stereological counting procedure. Specific fluorescence from tissue labeled in histological sections was captured by confocal microscopy with a constant exposure time across all images. From the quantification and stereological counting procedure, 16-bit image sections were analyzed using NIH Image J software (National Institutes of Health, Bethesda, MD, USA). Fluorescence intensity of pixel gray values in eight separate regions of interest per region of the normal and tumor tissues was calculated and averaged across each tissue region. This was performed separately for each label (NRP1 and DAPI). The fluorescence intensity for NRP1 in normal and tumor tissues was then compared using analysis of variance and Tukey's and Sidak's comparison tests.

Western blotanalysis.Protein lysates were derived from the tumor specimens and normal ovarian epithelial specimens via lysis in buffer containing protease inhibitors (Promega Corporation, Madison, WI, USA). Equal quantities of protein were separated by $10 \%$ sulfate polyacrylamide gel electrophoresis and then transferred onto a polyvinylidene fluoride membrane. Membranes were blocked with $5 \%$ non-fat milk in Tris-buffered saline containing $0.1 \%$ Tween-20 (TBST) for $2 \mathrm{~h}$ to avoid nonspecific binding. Following incubation with the primary antibodies overnight at $4^{\circ} \mathrm{C}$ (rabbit monoclonal NRP1 primary antibody; 1:200; Santa Cruz Biotechnology, Inc.) or rabbit polyclonal anti- $\beta$-actin (1:2,000; cat. no. A2668; Sigma-Aldrich), membranes were washed three times in TBST for $5 \mathrm{~min}$ and subsequently incubated with horseradish peroxidase-conjugated goat polyclonal anti-rabbit secondary antibody (1:1,000 dilution; cat. no. A0545; Sigma-Aldrich) for $2 \mathrm{~h}$ at room temperature. Signals were detected using enhanced chemiluminescence (GE Healthcare, Piscataway, NJ, USA) followed by film development.

Expression analysis by reverse transcription quantitative polymerase chain reaction ( $R T-q P C R)$. The mRNA expression of NRP1 was analyzed by qPCR. Total RNAs were extracted using TRIzol (Gibco-BRL, Carlsbad, CA, USA). qPCR was performed using Hot Start-IT SYBR Green qPCR Master mix (2X; USB Corporation, Cleveland, OH, USA). According to the manufacturer's instructions of the Hot Start-IT, $2 \mu \mathrm{g}$ of RNA was used as a template and RT-PCR was performed with $25 \mu \mathrm{l}$ Master mix. RT-PCR experiments were performed in a Light Cycler 480 system (Roche Applied Sciences, Indianapolis, IN, USA). Cycling parameters were set as follows: Hot start at $95^{\circ} \mathrm{C}$ for $10 \mathrm{~min}, 40$ cycles of amplification/quantification at $95^{\circ} \mathrm{C}$ for $10 \mathrm{sec}, 60^{\circ} \mathrm{C}$ for $30 \mathrm{sec}$ and $72^{\circ} \mathrm{C}$ for $30 \mathrm{sec}$. Melting curve analysis was performed using continuous fluorescence acquisition from $65-97^{\circ} \mathrm{C}$. These cycling parameters generated single amplicons for the two primer sets used according to the presence of a single melt peak. GAPDH was selected as the internal reference. All qPCRs were repeated three times for each gene and each sample was performed in triplicate. The primer sequences were as follows: NRP1, forward 5'-AAAACGGTGCCATCCCT-3' and reverse 5'-AAGAAGCAGAGTGGGTCGTT-3'. The 

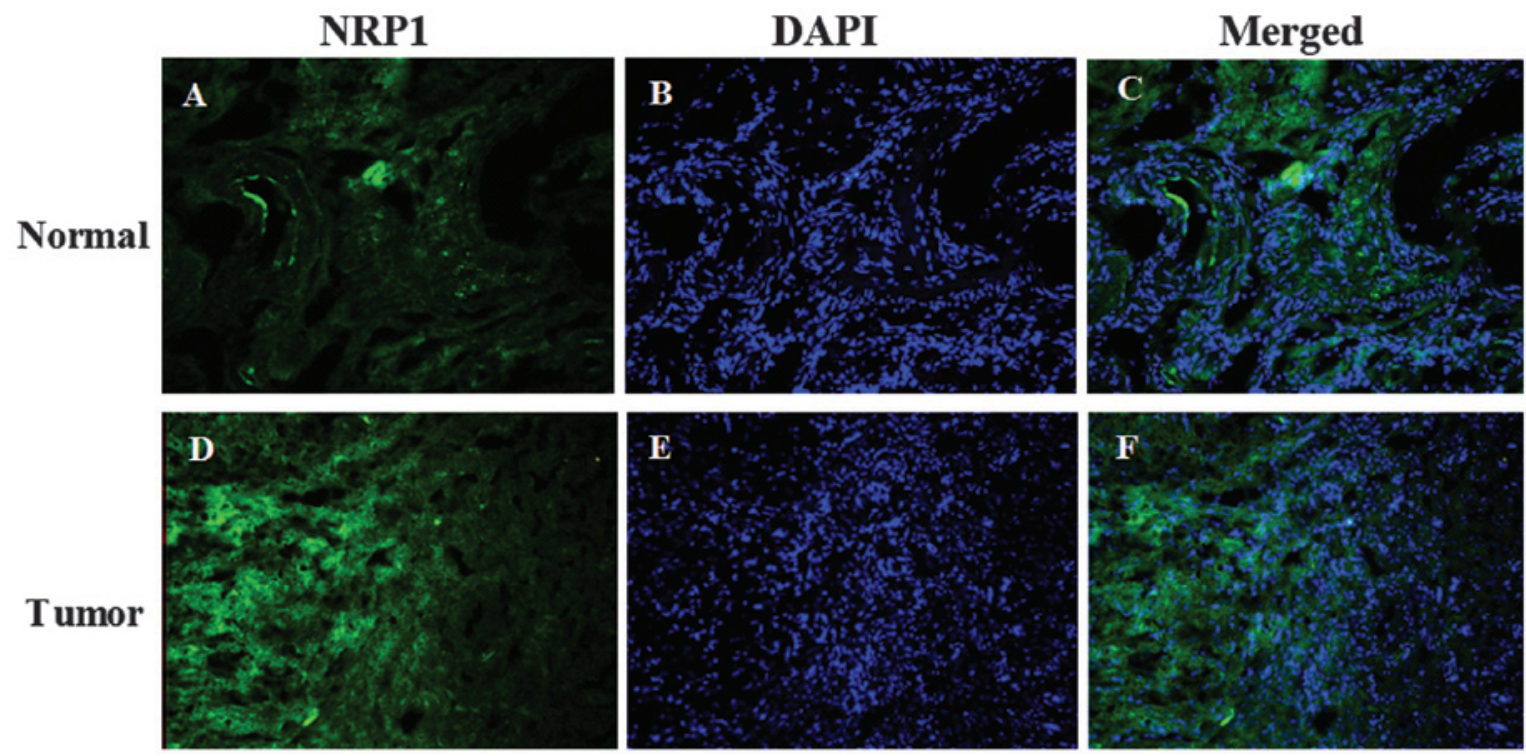

Figure 1. (A) Expression of NRP1 in normal tissues; (B) DAPI in normal tissues; (C) combination of (A and B); (D) the expression of NRP1 in tumor tissues; (E) DAPI in tumor tissues; (F) combination of (D and E). The protein was differentially expressed proteins in normal ovarian epithelium and epithelial ovarian carcinoma, respectively. The color intensity indicates the degree of protein regulation. NRP1, neuropilin 1; DAPI, 4',6-diamidino-2-phenylindole.

relative alterations in gene expression data were analyzed by the $2^{-\Delta \Delta C T}$ method. Triplicates were run for each sample in three independent experiments.

Clinicopathological analysis and postoperative follow up. Pathological analysis was performed by the Department of Pathology of Nantong University and validated by qualified experts assigned to the study. During the follow-up period, overall survival time was measured from diagnosis to mortality or to the last follow up (at 5 years). At the time of analysis, 86 patients (68.8\%) had succumbed to the disease, 37 patients $(29.6 \%)$ were alive and 2 patients were not identified at follow up. The estimated median survival time for all patients was 28 months and the calculated survival rates were $72.8 \%$ at 1 year, $48.0 \%$ at 2 years and $29.6 \%$ at 5 years. Following surgery, each patient was scheduled for a follow-up examination, including physical examination, complete blood count, tumor marker tests as well as an ultrasound scan of the pelvis every 3 months in the first year, semi-annually in the second year and annually after 3 years. More frequent examinations were scheduled if clinically indicated. The cause of mortality was registered and classified as mortality due to this cancer, other causes or unknown causes. During the follow-up period, overall survival was measured from diagnosis to mortality or to the last follow-up (at 5 years). Patient mortality was ascertained by reporting from the family and verified by a review of public records. For overall survival, the median follow-up of the surviving patients was 28 months (range 2-60 months).

Statistical analysis. Tukey's and Sidak's comparison tests were used to compare the fluorescence intensity. SPSS 19.0 statistical software (SPSS, Inc., Chicago, IL, USA) was adopted for data analysis. The $\chi^{2}$ test was used for comparisons between groups. Survival analysis was calculated by means of the Kaplan-Meier method and significant levels were assessed by means of the log-rank test. The results are

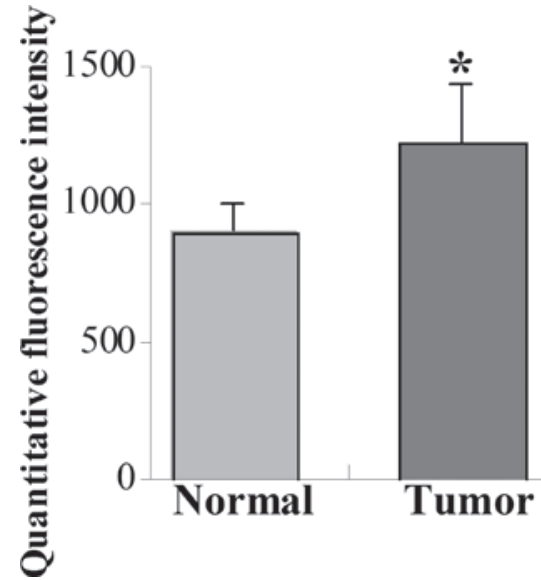

Figure 2. Quantitative fluorescence intensity of normal ovarian epithelium and epithelial ovarian carcinoma. ${ }^{*} \mathrm{P}<0.05$, compared with normal tissue.

expressed as the mean \pm standard deviation of at least three independent experiments. For all statistical analyses, $\mathrm{P}<0.05$ was considered to indicate a statistically significant difference.

\section{Results}

Immunofluorescence staining shows that Nrpl protein is present at a higher level in tumor tissue than in normal ovarian epithelium. NRP1 was detected primarily in the nucleus and cytoplasm of the normal ovarian epithelium (Fig. 1A and C). Compared with the normal ovarian epithelium, the EOC specimens demonstrated a stronger fluorescence intensity (Fig. 1). In addition, the quantitative fluorescence intensity of NRP1 was higher in tumor tissue than in normal specimens (Fig. 2). The expression of NRP1 was significantly upregulated in the tumor tissues compared with the normal tissues $(\mathrm{P}<0.001$; Figs. 1 and 2). 

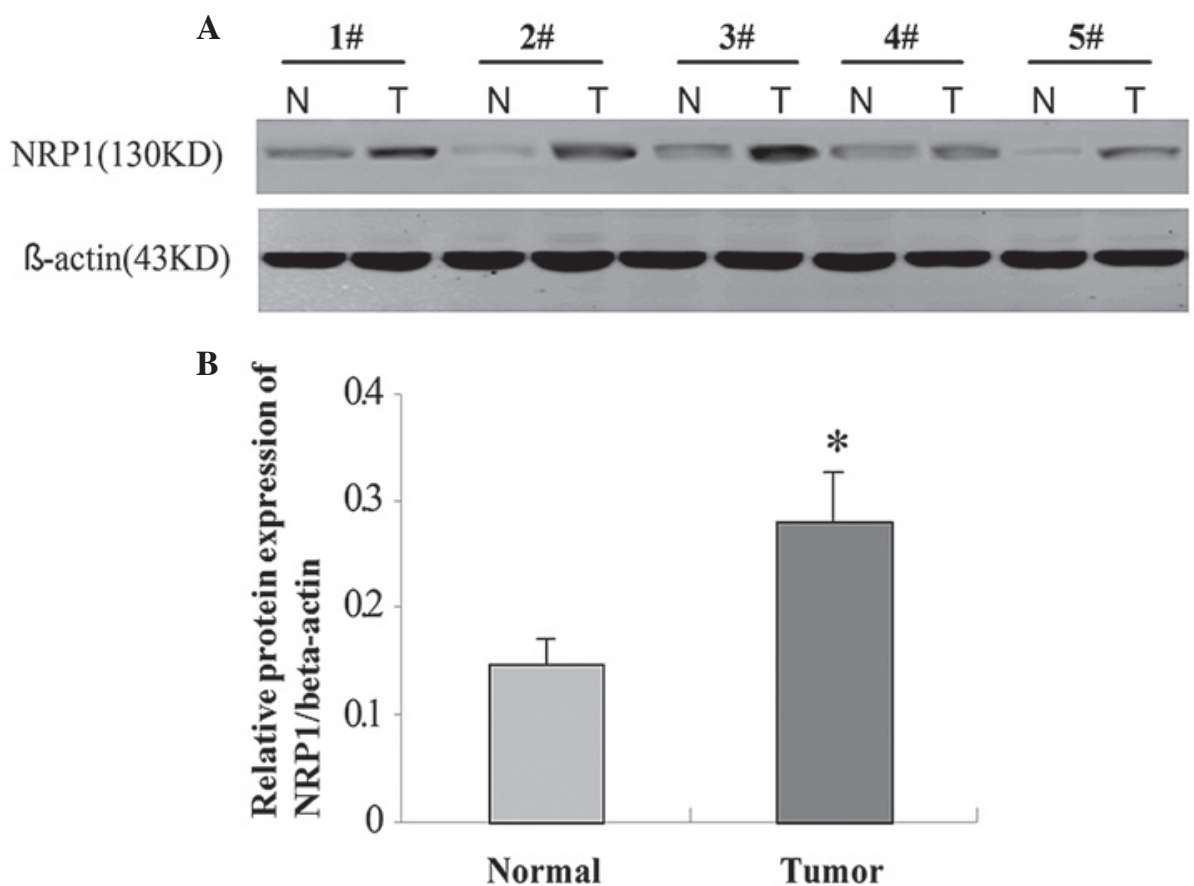

Figure 3. Protein expression of NRP1 in normal ovarian epithelium and epithelial ovarian carcinoma (n=5). (A) Representative results of NRP1 protein expression. (B) Relative NRP1 protein expression levels were markedly increased in tumor tissues compared with the normal specimens. NRP1, neuropilin 1; $\mathrm{N}$, normal; $\mathrm{T}$, tumor. ${ }^{*} \mathrm{P}<0.05$, compared with normal tissue.

Nrpl protein is present at a higher level in tumor tissue than in normal ovarian epithelium as shown by the results of the western blotting. The protein expression of NRP1 in ovarian tumor epithelium and normal ovarian epithelium was examined by western blot analysis. The relative quantity of NRP1 protein expression was normalized to $\beta$-actin. Five pairs of carcinoma and normal tissues were randomly selected and presented in Fig. 3A. The results demonstrated a band for NRP1 at $130 \mathrm{kDa}$ (Fig. 3A). The protein expression intensities of NRP1 were measured by densitometry (Fig. 3B). It was identified that the protein expression of NRP1 was upregulated in the majority of the ovarian tumor samples compared with normal tissues (Fig. 3A), while in a small number of cases (4\# in Fig. 3) the expression of NRP1 in the tumor tissues was similar to that found in the normal tissue (Fig. 3A). The average protein level of NRP1 in EOC was significantly higher than that in normal ovarian epithelial tissues $(\mathrm{P}<0.05$; Fig. $3 \mathrm{~B})$.

NRP1 mRNA level is higher in cancerous tissue compared with in normal epithelium. RT-qPCR was also performed to detect the mRNA expression of NRP1 in EOC and normal ovarian epithelial tissues to determine whether there is also an upregulation at the mRNA level. As shown in Table I, the mRNA expression of NRP1 was significantly higher in ovarian cancerous samples (median 474 copies/ $\mu 1$, range between 193 and 841 copies $/ \mu 1$ ) compared with that in normal samples (median 232 copies $/ \mu 1$, range between 102 and 314 copies $/ \mu 1$; $\mathrm{P}=0.008$ ). Quantification of the mRNA expression of NRP1 evaluated a 2.3 -fold increase in cancerous compared with normal tissues (Fig. 4).

Expression of NRPI is associated with pathological features of ovarian tumors. Correlations between RT-qPCR results

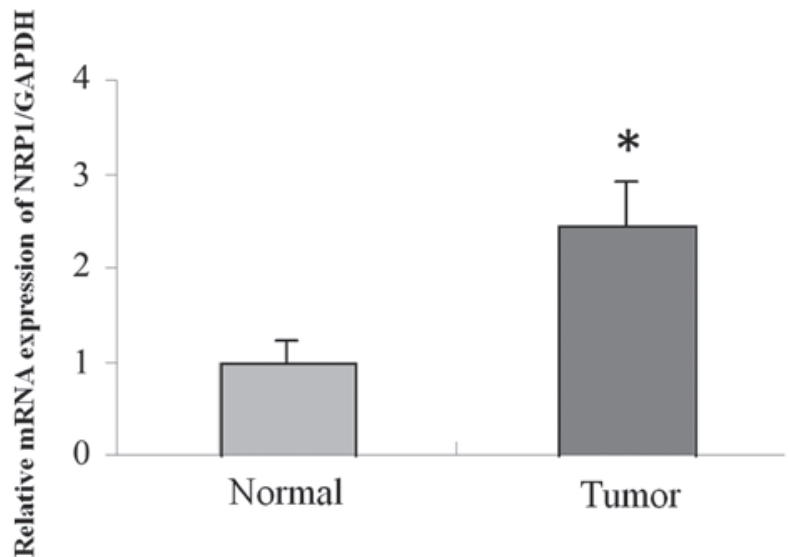

Figure 4. Quantitative polymerase chain reaction analysis of NRP1 expression in normal ovarian epithelium and epithelial ovarian carcinoma. The relative mRNA expression of NRP1 was significantly higher in tumor tissues compared with the normal specimens. NRP1, neuropilin 1 . " $\mathrm{P}<0.05$, compared with normal tissue.

of NRP1 expression in ovarian tumor tissues and various clinicopathological characteristics of patients were analyzed by $\chi^{2}$ test and listed in Table II. Using the quartile limits of mRNA expression to divide patient population into negative and positive producers allowed us to set the interquartile range (IQR) as a cut-off and to establish a significant correlation between mRNA expression and clinicopathological features. The median expression of NRP1 in cancerous tissues was 474 copies/ $\mu$ l. The samples were divided into the following two groups: The negative expression group of NRP1 $(\leq 474$ copies $/ \mu 1)$ and the positive expression group of NRP1 (>474 copies/ $\mu 1$ ). The upregulation of NRP1 significantly correlated with FIGO stage, histological grade, lymphatic 
Table I. mRNA expression of NRP1 in normal ovarian epithelium and epithelial ovarian carcinoma.

\begin{tabular}{lccc}
\hline Gene & $\begin{array}{c}\text { Normal } \\
(\text { copies } / \mu \text { l median })\end{array}$ & $\begin{array}{c}\text { Tumor } \\
(\text { copies/ } \mu 1 \text { median })\end{array}$ & P-value \\
\hline NRP1 & $232(102-314)$ & $474(193-841)$ & 0.008 \\
\hline
\end{tabular}

NRP1, neuropilin 1 .

Table II. Association between NRP1 expression levels in epithelial ovarian carcinoma and clinicopathological features.

\begin{tabular}{|c|c|c|c|c|}
\hline \multirow[b]{2}{*}{ Characteristic } & \multirow[b]{2}{*}{ No. } & \multicolumn{3}{|c|}{ NRP1 } \\
\hline & & $\mathrm{N}$ & $\mathrm{P}$ & P-value \\
\hline Age (years) & & & & 0.102 \\
\hline$\leq 59$ & 63 & 23 & 40 & \\
\hline$>59$ & 62 & 18 & 44 & \\
\hline Tumor size $(\mathrm{cm})$ & & & & 0.105 \\
\hline$\leq 2$ & 45 & 17 & 28 & \\
\hline$>2$ & 80 & 24 & 56 & \\
\hline FIGO stage & & & & 0.025 \\
\hline $\mathrm{I} / \mathrm{II}$ & 59 & 16 & 43 & \\
\hline III/IV & 72 & 31 & 41 & \\
\hline Histological grade & & & & 0.049 \\
\hline Well differentiated & 55 & 22 & 33 & \\
\hline Poorly differentiated & 70 & 19 & 51 & \\
\hline Histotype & & & & 0.395 \\
\hline Serous & 58 & 18 & 40 & \\
\hline Endometrioid & 49 & 15 & 34 & \\
\hline Clear cell & 13 & 5 & 8 & \\
\hline Mucinous & 3 & 2 & 1 & \\
\hline Undifferentiated & 1 & 0 & 1 & \\
\hline Other & 1 & 1 & 0 & \\
\hline Lymphatic metastasis & & & & 0.006 \\
\hline Negative & 53 & 24 & 29 & \\
\hline Positive & 72 & 17 & 55 & \\
\hline Distant metastasis & & & & 0.024 \\
\hline Negative & 67 & 23 & 44 & \\
\hline Positive & 58 & 18 & 40 & \\
\hline
\end{tabular}

No., number of patients; N, negative; $\mathrm{P}$, positive; NRP1, neuropilin 1; FIGO, International Federation of Gynecology and Obstetrics staging.

metastasis and distant metastasis ( $\mathrm{P}<0.05$, respectively). However, no significant correlation between NRP1 expression and age, tumor size and histological type was identified ( $\mathrm{P}>0.05$; Table II).

NRP1 expression is associated with patient survival rate. The prognostic effect of NRP1 on the overall survival rate of ovarian carcinoma patients was investigated by comparing the

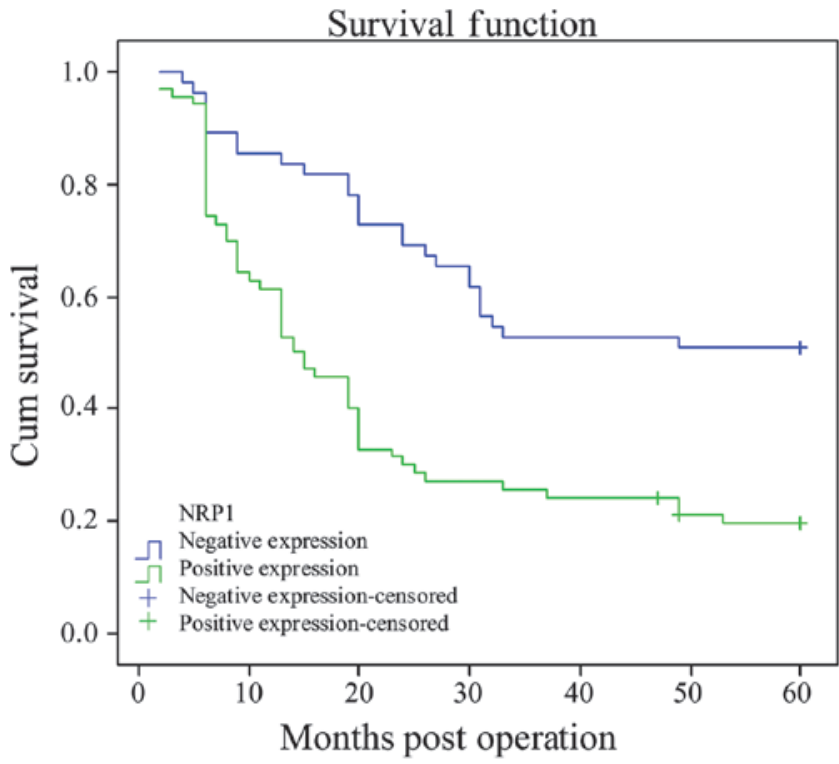

Figure 5. Kaplan-Meier survival curves of cancer patients following surgical resection. The cancer patients were divided into negative expression and positive expression groups of NRP1. A positive expression of NRP1 was strongly associated with a poorer patient survival time. NRP1, neuropilin 1. Negative expression-censored, patients with NRP1 negative expression lost to follow-up; positive expression-censored, patients with NRP1 positive expression lost to follow-up.

5-year survival rate of patients with tumors expressing NRP1 using Kaplan-Meier survival curves and the log-rank test. There were 70 cases in the positive NRP1 expression group ( $>474$ copies $/ \mu 1$ ), of which 58 cases succumbed to ovarian carcinoma, two cases were not identified at follow up and the 5-year overall survival rate was $14.3 \%$. For the negative NRP1 expression group ( $<474$ copies $/ \mu 1)$, there were 55 cases, of which 28 cases succumbed to the disease and the 5-year overall survival rate was $49.1 \%$. It was found that the overall survival time of the positive NRP1 expression group was significantly shorter than that of the negative NRP1 expression group ( $\mathrm{P}<0.001$; Fig. 5).

\section{Discussion}

In the present study, NRP1 was found to be extensively upregulated in EOC compared with normal ovarian epithelium. Immunofluorescence, western blot analysis and RT-qPCR were used to detect the expression of NRP1 in ovarian cancerous surgical specimens and normal ovarian epithelial tissues. The mRNA and protein expression of NRP1 in ovarian epithelial cancer tissues was significantly higher than that in normal ovarian epithelial tissues. It was found that the overall survival time of the high NRP1 expression group was significantly shorter than that of the low NRP1 expression group $(\mathrm{P}<0.05)$. The changes in NRP1 expression in ovarian cancer in the present study indicates an important role of NRP1 in the development of EOC. In addition, a higher expression of NRP1 correlated with a poor prognosis in ovarian tumors, demonstrating that NRP1 may act as a promoter in ovarian epithelial cancer.

NRP1 is a transmembrane glycoprotein that acts as a co-receptor for a number of extracellular ligands, including 
class III/IV semaphorins, certain isoforms of VEGF and transforming growth factor- $\beta$. NRP1 is expressed at high levels in several tumor cells, where it has been implicated in cell migration and survival $(19,20)$. An exact understanding of the role of NRP1 in the immune system has been obscured by the differences in NRP1 expression observed between mice and humans. In mice, NRP1 is selectively expressed on thymus-derived $\mathrm{T}$ regulatory cells (Tregs) and markedly enhances immunosuppressive functions. In humans, NRP1 is expressed on plasmacytoid dendritic cells (pDCs) where it aids in priming immune responses and on a subset of Tregs isolated from secondary lymph nodes. Preliminary studies show that NRP1 expression on $T$ cells confers enhanced immunosuppressive activity $(21,22)$. However, the underlying mechanism remains to be elucidated. The expression of NRP1 has also been identified in activated $\mathrm{T}$ cells and Tregs isolated from inflammatory microenvironments, suggesting that NRP1 may be a novel $\mathrm{T}$ cell activation marker (23). Of clinical interest, NRP1 may enhance tumor infiltration of Tregs and a decrease in NRP1+Tregs correlates with successful chemotherapy, suggesting a specific role for NRP1 in cancer pathology (21). As a therapeutic target, NRP1 allows simultaneous targeting of NRP1-expressing tumor vasculature, NRP1+Tregs and pDCs. With the development of anti-NRP1 monoclonal antibodies and cell-penetrating peptides, NRP1 represents a promising new target for cancer therapies (24).

NRP1 has been implicated as a tumor suppressor in other types of cancer (25). NRP1 and NRP2 are transmembrane glycoproteins, which interact with VEGF to prevent tumor cell apoptosis and regulate angiogenesis. For example, in colorectal cancer, an increased expression of NRP1 and NRP2 in epithelium as well as an increased expression of NRP1 in vessels may be associated with the progression of colorectal cancer (19). A more recent study on miRNAs demonstrated that the downregulation of miR-320, which regulates NRP1, in blood vessels is inversely correlated with vascularity in oral squamous cell carcinoma (OSCC) tissues (26). By administering either miR-320 precursor or antagonist, miR-320 suppressed the migration, adhesion and tube formation of vascular endothelial cells. Knockdown of NRP1 reduced antagomiR-320-induced cell migration (26). Additionally, miR-320 expression was demonstrated to be regulated by hypoxia in growth factor-deficient conditions by hypoxia-inducible factor-1 $\alpha$ (27). Furthermore, a lentivirus carrying the miR-320 precursor suppressed the tumorigenicity of OSCC cells and tumor angiogenesis in vivo (28). These data show that miR-320 may regulate the function of vascular endothelial cells by targeting NRP1 and have the potential to be developed as an anti-angiogenic or anti-cancer drug. These results taken together with the results of the present study demonstrate that NRP1 may be associated with cancer progression in EOC.

Although the present study provided valuable results regarding the role of NRP1 in EOC, a limitation of the study was that only 15 cases of normal tissues were used. A larger number of cases should be used in future studies. The present study attempted to use double-labeling immunofluorescence staining and confocal microscopy instead of immunohistochemistry. It was difficult to control the chromogenic time in immunohistochemistry and the sensitivity of staining was lower than that of immunofluorescence. In addition, the immunohistochemistry results could be affected by numerous artificial factors $(24,25)$. By contrast, fluorescence could objectively reflect the expression of the protein. The main advantages of this technology included its strong specificity, high sensitivity and time efficiency.

In the present study, quantitative mRNA expression was used to analyze the association of NRP1 with the clinicopathological factors of EOC as well as the overall survival rate. Using the quartile limits of mRNA expression to divide the patient population into low and high producers, this allowed us to set IQR as a cut-off and to establish a significant correlation between mRNA expression and clinicopathological factors as well as survival rate, which may more accurately reflect the real situation.

In conclusion, in the present study, NRP1 was found to be overexpressed in human EOC. A higher expression of NRP1 was strongly associated with a poorer patient survival time. This may provide a novel prognostic method and a promising treatment strategy for EOC.

\section{References}

1. Paes MF, Daltoé RD, Madeira KP, et al: A retrospective analysis of clinicopathological and prognostic characteristics of ovarian tumors in the state of Espírito Santo, Brazil. J Ovarian Res 4: 14, 2011.

2. Siegel R, Naishadham D and Jemal A: Cancer statistics, 2012. CA Cancer J Clin 62: 10-29, 2012.

3. Hudson LG, Zeineldin R and Stack MS: Phenotypic plasticity of neoplastic ovarian epithelium: unique cadherin profiles in tumor progression. Clin Exp Metastasis 25: 643-655, 2008.

4. Domenighetti G,Moccia A and Gayer R: Observational case-control study of non-invasive ventilation in patients with ARDS. Monaldi Arch Chest Dis 69: 5-10, 2008.

5. Teoh D and Secord AA: Antiangiogenic agents in combination with chemotherapy for the treatment of epithelial ovarian cancer. Int J Gynecol Cancer 22: 348-359, 2012.

6. Usha L, Sill MW, Darcy KM, et al: A Gynecologic Oncology Group phase II trial of the protein kinase C-beta inhibitor, enzastaurin and evaluation of markers with potential predictive and prognostic value in persistent or recurrent epithelial ovarian and primary peritoneal malignancies. Gynecol Oncol 121: 455-461, 2011.

7. Wu H, Yao L, Mei J and Li F: Development of synthetic of peptide-functionalized liposome for enhanced targeted ovarian carcinoma therapy. Int J Clin Exp Pathol 8: 207-216, 2015.

8. Kannan K, Coarfa C, Chao PW, et al: Recurrent BCAM-AKT2 fusion gene leads to a constitutively activated AKT2 fusion kinase in high-grade serous ovarian carcinoma. Proc Natl Acad Sci USA 112: E1272-E1277, 2015.

9. Gurler H, Yu Y, Choi J, Kajdacsy-Balla AA and Barbolina MV: Three-dimensional collagen type I matrix up-regulates nuclear isoforms of the microtubule associated protein tau implicated in resistance to paclitaxel therapy in ovarian carcinoma. Int J Mol Sci 16: 3419-3433, 2015.

10. Morris LG, Veeriah S and Chan TA: Genetic determinants at the interface of cancer and neurodegenerative disease. Oncogene 29: 3453-3464, 2010.

11. Gan GN, Weickhardt AJ, Scheier B, et al: Stereotactic radiation therapy can safely and durably control sites of extra-central nervous system oligoprogressive disease in anaplastic lymphoma kinase-positive lung cancer patients receiving crizotinib. Int J Radiat Oncol Biol Phys 88: 892-898, 2014.

12. Catalano A, Caprari P, Rodilossi S, et al: Cross-talk between vascular endothelial growth factor and semaphorin-3A pathway in the regulation of normal and malignant mesothelial cell proliferation. FASEB J 18: 358-360, 2004.

13. Cao L, Liu X, Lin EJ, et al: Environmental and genetic activation of a brain-adipocyte BDNF/leptin axis causes cancer remission and inhibition. Cell 142: 52-64, 2010.

14. Casazza A, Fu X, Johansson I, et al: Systemic and targeted delivery of semaphorin $3 \mathrm{~A}$ inhibits tumor angiogenesis and progression in mouse tumor models. Arterioscler Thromb Vasc Biol 31: 741-749, 2011. 
15. Maione F, Molla F, Meda C, et al: Semaphorin 3A is an endogenous angiogenesis inhibitor that blocks tumor growth and normalizes tumor vasculature in transgenic mouse models. J Clin Invest 119: 3356-3372, 2009

16. Sakurai A, Gavard J, Annas-Linhares Y, et al: Semaphorin 3E initiates antiangiogenic signaling through plexin D1 by regulating Arf6 and R-Ras. Mol Cell Biol 30: 3086-3098, 2010.

17. Varshavsky A, Kessler O, Abramovitch S, et al: Semaphorin-3B is an angiogenesis inhibitor that is inactivated by furin-like pro-protein convertases. Cancer Res 68: 6922-6931, 2008.

18. Zheng C, Zhou Q, Wu F, et al: Semaphorin3F down-regulates the expression of integrin alpha(v)beta3 and sensitizes multicellular tumor spheroids to chemotherapy via the neuropilin-2 receptor in vitro. Chemotherapy 55: 344-352, 2009.

19. Staton CA, Koay I, Wu JM, Hoh L, Reed MW and Brown NJ: Neuropilin-1 and neuropilin-2 expression in the adenoma-carcinoma sequence of colorectal cancer. Histopathology 62: 908-915, 2013.

20. Jia H, Cheng L, Tickner M, Bagherzadeh A, Selwood D and Zachary I: Neuropilin-1 antagonism in human carcinoma cells inhibits migration and enhances chemosensitivity. Br J Cancer 102: 541-552, 2010.

21. Chaudhary B, Khaled YS, Ammori BJ and Elkord E: Neuropilin 1: function and therapeutic potential in cancer. Cancer Immunol Immunother 63: 81-99, 2014.
22. Bruder D, Probst-Kepper M, Westendorf AM, et al: Neuropilin-1: A surface marker of regulatory $\mathrm{T}$ cells. Eur J Immunol 34: 623-630, 2004

23. Renand A, Milpied P, Rossignol J, et al: Neuropilin-1 expression characterizes T follicular helper (Tfh) cells activated during B cell differentiation in human secondary lymphoid organs. PLoS One 8: e85589, 2013.

24. Chaudhary B, Khaled YS, Ammori BJ and Elkord E: Neuropilin 1 function and therapeutic potential in cancer. Cancer Immunol Immunother 63: 81-99, 2014.

25. Kamiya T, Kawakami T, Abe Y, et al: The preserved expression of neuropilin (NRP) 1 contributes to a better prognosis in colon cancer. Oncol Rep 15: 369-373, 2006.

26. Wu YY, Chen YL, Jao YC, et al: miR-320 regulates tumor angiogenesis driven by vascular endothelial cells in oral cancer by silencing neuropilin 1. Angiogenesis 17: 247-260, 2014.

27. Wan LY, Deng J, Xiang XJ, Zhang L, et al: miR-320 enhances the sensitivity of human colon cancer cells to chemoradiotherapy in vitro by targeting FOXM1. Biochem Biophys Res Commun 457: 125-32, 2015.

28. Wu YY, Chen YL, Jao YC, Hsieh IS, Chang KC and Hong TM: miR-320 regulates tumor angiogenesis driven by vascular endothelial cells in oral cancer by silencing neuropilin 1. Angiogenesis 17: 247-260, 2014. 